\title{
Erratum to: Risk factors for relapse and long-term outcome in steroid-dependent nephrotic syndrome treated with rituximab
}

\author{
Koichi Kamei $^{1} \cdot$ Masao Ogura $^{1} \cdot$ Mai Sato $^{1} \cdot$ Mayumi Sako $^{2} \cdot$ Kazumoto Iijima $^{3}$. \\ Shuichi Ito ${ }^{1,4}$
}

Published online: 21 September 2015

(C) IPNA 2015

\section{Erratum to: Pediatr Nephrol}

DOI 10.1007/s00467-015-3197-0

In the Results subsection of the abstract, the sentence "Fifty percent relapse-free survival in patients without a history of SRNS was 615 days, longer than that of patients with one relapse (393 days) $(p=0.005)$." should have read "Fifty percent relapse-free survival in patients without a history of SRNS was 615 days, longer than that of patients with a history of SRNS (393 days) ( $p=0.005)$.”. The authors apologize for this error and any inconvenience caused.

The online version of the original article can be found at http://dx.doi.org/ 10.1007/s00467-015-3197-0.

Shuichi Ito

itoshu@yokohama-cu.ac.jp

1 Division of Nephrology and Rheumatology, National Center for Child Health and Development, Tokyo, Japan

2 Division for Clinical Trials, Department of Development Strategy, Center for Social and Clinical Research, National Research Institute for Child Health and Development, National Center for Child Health and Development, Tokyo, Japan

3 Department of Pediatrics, Kobe University Graduate School of Medicine, Hyogo, Japan

4 Department of Pediatrics, Yokohama City University, 3-9, Fukuura, Kanazawa-ku, Yokohama-shi, Kanagawa 236-0004, Japan 